Benedetto Bongiorno and Luisa Di Piazza* Department of Mathematics, University of Palermo, Via Archirafi 34, 90123 Palermo, Italy

Valentin Skvortsov ${ }^{\dagger}$ Department of Mathematics, Moscow State University, Moscow 119899, Russia

\title{
ON CONTINUOUS MAJOR AND MINOR FUNCTIONS FOR THE $n$-DIMENSIONAL PERRON INTEGRAL ${ }^{\ddagger}$
}

\begin{abstract}
We prove that the $n$-dimensional Perron integral with respect to the full interval basis, without any regularity condition, defined by continuous major and minor functions is equivalent to the one defined by major and minor functions which are not supposed to be continuous.
\end{abstract}

\section{Introduction}

It is well known (see for example [7]) that, for any basis of differentiation, the Henstock-Kurzweil integral ( $H K$-integral) is equivalent to the Perron integral ( $P$-integral) defined in terms of the same basis. But this equivalence is related to the definition of the Perron integral in which major and minor functions are not supposed to be continuous. The question of whether we get an equivalent definition by taking into account only continuous (with respect to the basis) major and minor functions is still open in the general case (see [2, p. 202] and $[3])$.

In the case of the one-dimensional Perron integral this equivalence is proved by several methods (see [5], [8], [9]). In the multidimensional case the equivalence was recently established (see [6]) for the basis of regular intervals, using

\footnotetext{
Key Words: Perron integral, basis of differentiation, variation

Mathematical Reviews subject classification: Primary: 26A39, 26A42, 26A45

Received by the editors June 11, 1996

‡Dedicated to Professor Washek Pfeffer on his birthday

*Supported by M.U.R.S.T. of Italy and by INTAS-94-1044.

† Supported by C.N.R. of Italy and by INTAS-94-1044.
} 
the fact that any indefinite $P$-integral is differentiable with respect to this basis almost everywhere. Here we extend this result to the case of the $P$-integral and the $H K$-integral defined with respect to the full interval basis, without any regularity condition. As the above mentioned result on differentiability is not available for the full basis (see [4, p. 95]), we are to employ some other technique here.

\section{Preliminaries}

We recall some definitions and notations. The $n$-dimensional Lebesgue measure of a set $E \subset \mathbb{R}^{n}$ is denoted by $|E|$. In $\mathbb{R}^{n}$ we shall use the norm $\|x\|=\max _{1 \leq i \leq n}\left|x_{i}\right|$, where $x=\left(x_{1}, x_{2}, \ldots, x_{n}\right)$. Then the $\delta$-neighborhood of $x$, denoted by $U(x, \delta)$, is an open cube centered at $x$ with side equal to $2 \delta$.

An interval in $\mathbb{R}^{n}$ is a set $I=\left[a_{1}, b_{1}\right] \times\left[a_{2}, b_{2}\right] \times \cdots \times\left[a_{n}, b_{n}\right]$ with $a_{i}<b_{i}$, $i=1,2, \ldots, n$. Throughout this paper $\Delta$ denotes a fixed interval and $\mathcal{I}$ the family of all subintervals of $\Delta$.

A basis of differentiation (or simply a basis) on $\Delta$ is, by definition, any subset $\mathcal{B}$ of $\mathcal{I} \times \Delta$ such that $(I, x) \in \mathcal{B}$ implies $x \in I$.

Given a basis $\mathcal{B}$, an interval $I$ is called a $\mathcal{B}$-interval if $(I, x) \in \mathcal{B}$, for some $x \in I$.

For a set $E \subset \Delta$ we write

$$
\mathcal{B}(E)=\{(I, x) \in \mathcal{B}: I \subset E\} \text { and } \mathcal{B}[E]=\{(I, x) \in \mathcal{B}: x \in E\} .
$$

If a function $\delta(x)>0$ is defined on $\Delta$ we denote

$$
\mathcal{B}_{\delta}=\{(I, x) \in \mathcal{B}: I \subset U(x, \delta(x))\} .
$$

Note that $\mathcal{B}_{\delta}$ is also a basis on $\Delta$. So the meaning of $\mathcal{B}_{\delta}(E)$ and $\mathcal{B}_{\delta}[E]$ is clear from (1) and (2).

We say that a basis $\mathcal{B}$ is a Vitali basis, if for any $x$ and for any $\delta$ the set $\mathcal{B}_{\delta}[\{x\}]$ is nonempty.

We say that a basis $\mathcal{B}$ is a free-point basis if for any $\mathcal{B}$-interval $I$ and for any $x \in I$ we have $(I, x) \in \mathcal{B}$.

We say that a basis $\mathcal{B}$ is a full basis if for each interval $I \in \mathcal{I}$ and for each $x \in I$ we have $(I, x) \in \mathcal{B}[\{x\}]$ (in [10] the term "full covering relation" is used for a similar notion).

Our main results are related to the full basis which we denote by $\mathcal{F}$. Clearly the full basis $\mathcal{F}$ is a free-point Vitali basis. Note that in our notation any interval $I \in \mathcal{I}$ is an $\mathcal{F}$-interval.

A finite subset $\pi$ of $\mathcal{B}[E]$ is called a partition on $E$ if for distinct pairs $\left(I^{\prime}, x^{\prime}\right)$ and $\left(I^{\prime \prime}, x^{\prime \prime}\right)$ in $\pi$, the intervals $I^{\prime}$ and $I^{\prime \prime}$ are nonoverlapping. 
Given an interval function $F$ defined on the family of all $\mathcal{B}$-intervals, a set $E \subset \Delta$ and a function $\delta(x)>0$ we denote for any interval $A \in \mathcal{I}$

$$
V_{\delta}(A)=V\left(\mathcal{B}_{\delta}, F, E, A\right)=\sup \left\{\sum_{(I, x) \in \pi}|F(I)|: \pi \subset \mathcal{B}_{\delta}[E] \cap \mathcal{B}_{\delta}(A)\right\}
$$

and we call it the $\delta$-variation over $\mathcal{B}$ of the function $F$ on $E \cap A$. If $E \cap A=\emptyset$, we define $V_{\delta}(A)=0$. Clearly, for each fixed $E$, the interval function $V_{\delta}(A)$ is non-negative and superadditive.

Let $\mathcal{B}$ be a Vitali basis. An interval function $F$ defined on the family of all $\mathcal{B}$-intervals is said to be $\mathcal{B}$-continuous at a point $x$ if for any $\epsilon>0$ there exists $\eta>0$ such that $|F(I)|<\epsilon$ whenever $(I, x) \in \mathcal{B}_{\eta}[\{x\}]$.

\section{The $\delta$-Variation and the Perron Integral}

The following result was established in [6] for some class of regular bases. We are proving here the same proposition for any free-point Vitali basis and then applying it to the full basis $\mathcal{F}$.

Proposition 1 Let $\mathcal{B}$ be a free-point Vitali basis and let $F$ be a $\mathcal{B}$-continuous additive function defined on the family of all $\mathcal{B}$-intervals. Then for a fixed set $E \subset \Delta$ and a fixed function $\delta(x)>0$ the interval function $V_{\delta}(I)=$ $V\left(\mathcal{B}_{\delta}, F, E, I\right)$ is $\mathcal{F}$-continuous at each point $x \in \Delta$.

Proof. The proof we are giving here is similar to that given in [6]. Let $x_{0} \in \Delta$ and let $\epsilon>0$. We have to prove that there exists $\eta>0$ such that $V_{\delta}(A)<\epsilon$ for each $A \in \mathcal{F}_{\eta}\left[\left\{x_{0}\right\}\right]$.

Since $F$ is $\mathcal{B}$-continuous we can choose $\eta_{1}>0$ such that

$$
|F(J)|<\frac{\epsilon}{2^{n+1}} \text { for any }\left(J, x_{0}\right) \in \mathcal{B}_{\eta_{1}}\left[\left\{x_{0}\right\}\right] .
$$

Let $K$ be the closed ball of radius $\eta_{2}<\eta_{1}$ centered in $x_{0}$, i.e. the closure of $U\left(x_{0}, \eta_{2}\right)$. Note that $K \subset U\left(x_{0}, \eta_{1}\right)$. Consider a partition

$$
\pi=\{(I, x)\} \subset \mathcal{B}_{\delta}[E \cap K] \cap \mathcal{B}_{\delta}(K)
$$

such that

$$
\sum_{\pi}|F(I)|>V_{\delta}(K)-\frac{\epsilon}{4}
$$


Now let $\eta>0$ be such that $\eta \leq \eta_{2}$ and the condition $U\left(x_{0}, \eta\right) \cap I \neq \emptyset$ implies $x_{0} \in I$, for each interval $I$ with $(I, x) \in \pi$. Let $J_{1}, J_{2}, \cdots, J_{k}$ be such intervals. Clearly

$$
k \leq 2^{n} .
$$

Since $x_{0} \in J_{i}$ and $J_{i} \subset K \subset U\left(x_{0}, \eta_{1}\right)$, we have $\left(J_{i}, x_{0}\right) \in \mathcal{B}_{\eta_{1}}\left[\left\{x_{0}\right\}\right]$. Then by (3)

$$
\left|F\left(J_{i}\right)\right|<\frac{\epsilon}{2^{n+1}}, \quad i=1,2, \cdots, k
$$

Let $\pi_{1}=\pi \backslash \cup_{i=1}^{k}\left(J_{i}, x_{0}\right)$. By (5) and (6)

$$
\sum_{\pi_{1}}|F(I)|>\sum_{\pi}|F(I)|-\frac{\epsilon}{2}
$$

Now let $A \in \mathcal{F}_{\eta}\left[\left\{x_{0}\right\}\right]$. We claim that $V_{\delta}(A)<\epsilon$. Indeed the condition $V_{\delta}(A) \geq$ $\epsilon$ for some $A$ implies the existence of a partition $\pi_{2} \subset \mathcal{B}_{\delta}[E \cap A] \cap \mathcal{B}_{\delta}(A)$ such that

$$
\sum_{\pi_{2}}|F(I)|>V_{\delta}(A)-\frac{\epsilon}{4} \geq \frac{3 \epsilon}{4}
$$

Note that $\pi_{1} \cup \pi_{2} \subset \mathcal{B}_{\delta}[E \cap K] \cap \mathcal{B}_{\delta}(K)$. Then, using (4), (7) and (8), we get the contradiction

$$
V_{\delta}(K) \geq \sum_{\pi_{1}}|F(I)|+\sum_{\pi_{2}}|F(I)|>V_{\delta}(K)-\frac{\epsilon}{4}-\frac{\epsilon}{2}+\frac{3 \epsilon}{4}=V_{\delta}(K) .
$$

Our next results are related to the case $\mathcal{B}=\mathcal{F}$.

The lower derivative of an interval function $F$ at a point $x$ with respect to the basis $\mathcal{F}$ is defined as

$$
\underline{D}_{\mathcal{F}} F(x)=\sup _{\delta} \inf \left\{\frac{F(I)}{|I|}:(I, x) \in \mathcal{F}_{\delta}[\{x\}]\right\} .
$$

The upper derivative of $F$ at a point $x$ with respect to the basis $\mathcal{F}$ is defined as

$$
\bar{D}_{\mathcal{F}} F(x)=\inf _{\delta} \sup \left\{\frac{F(I)}{|I|}:(I, x) \in \mathcal{F}_{\delta}[\{x\}]\right\} .
$$

If $\underline{D}_{\mathcal{F}} F(x)=\bar{D}_{\mathcal{F}} F(x) \neq \pm \infty$, we say that $F$ is $\mathcal{F}$-differentiable at $x$ and the derivative is denoted by $D_{\mathcal{F}} F(x)$ (this is called the strong derivative in [8]).

Let $f$ be a point function on $\Delta$. An interval function $M$ (resp. $m$ ) is called a $\mathcal{F}$-major (resp. $\mathcal{F}$-minor) function of $f$ on $\Delta$ if it is superadditive (resp. 
subadditive) and the lower (resp. upper) derivative with respect to $\mathcal{F}$ satisfies the inequality

$$
\underline{D}_{\mathcal{F}} M(x) \geq f(x) \quad\left(\operatorname{resp} . \bar{D}_{\mathcal{F}} m(x) \leq f(x)\right) \text { for all } x \in \Delta .
$$

A function $f$ is said to be $P$-integrable (resp. $P_{0}$-integrable) on $\Delta$ with respect to basis $\mathcal{F}$ if

$$
-\infty<\inf _{M}\{M(\Delta)\}=\sup _{m}\{m(\Delta)\}<\infty,
$$

where "inf" is taken over all $\mathcal{F}$-major functions (resp. $\mathcal{F}$-continuous $\mathcal{F}$-major functions) $M$ and "sup" is taken over all $\mathcal{F}$-minor functions (resp. $\mathcal{F}$-continuous $\mathcal{F}$-minor functions) $m$. The common value is denoted by

$$
(P) \int_{\Delta} f \quad\left(\text { resp. }\left(P_{0}\right) \int_{\Delta} f\right)
$$

and is called the $P$-integral (resp. $P_{0}$-integral) of $f$ over $\Delta$. Since $f$ is also integrable on each subinterval $I$ of $\Delta$ we can define $F(I)=(P) \int_{I} f$ (resp. $\left.=\left(P_{0}\right) \int_{I} f\right)$ to be the indefinite integral of $f$.

The $P$-integral is known (see [7]) to be equivalent to the classical $n$ dimensional Henstock-Kurzweil integral. Hence it is additive with respect to the $\mathcal{F}$-intervals, and $F$ is an additive $\mathcal{F}$-continuous interval function.

This last property is true also for the $P_{0}$-integral. Indeed let $J_{1}$ and $J_{2}$ be two adjacent intervals such that $J=J_{1} \cup J_{2}$ is also an interval. Then it is enough to note that if $M_{1}$ and $M_{2}$ are continuous $\mathcal{F}$-major (or $\mathcal{F}$-minor) functions of $f$ on $J_{1}$ and $J_{2}$, respectively, the function $M(I)=M_{1}\left(I \cap J_{1}\right)+$ $M_{2}\left(I \cap J_{2}\right)$ defined for any interval $I \subset J$ is a continuous $\mathcal{F}$-major (or $\mathcal{F}$-minor) function of $f$ on $J$.

We need the following result.

Proposition 2 If a function $f$ is $P$-integrable on an interval $I \in \mathcal{I}$ then, given a closed set $E \subset I$, there exists a non-empty portion of $E$ on which $f$ is Lebesgue integrable.

Proof. This assertion is proved in [1] for the case $E=I$. In fact almost the same proof is valid for the more general case we need here.

The following assertion is known as Saks-Henstock lemma for the $H K$-integral (see [7]). In view of the above mentioned equivalence of the $H K$-integral and the $P$-integral we formulate it for a $P$-integrable function. 
Proposition 3 If a function $f$ is $P$-integrable on an interval $I \in \mathcal{I}$ and $F$ is its indefinite $P$-integral, then for any $\epsilon>0$ there exists a function $\delta(x)$ such that for any partition $\pi$ on $I, \pi \in \mathcal{F}_{\delta}(I)$ we have

$$
\sum_{(x, J) \in \pi}|f(x)| J|-F(J)|<\epsilon
$$

Corollary 4 Let a function $f$ be P-integrable on $I \in \mathcal{I}$ with the indefinite integral $F$ and let $f(x)=0$ if $x \in T$ for some set $T \subset I$. Then for any $\epsilon>0$ there exists a function $\delta(x)>0$ defined on I such that

$$
V\left(\mathcal{F}_{\delta}, F, T, I\right)<\epsilon
$$

\section{The Main Result}

Now we can formulate and prove the main result of the paper. Since in the rest of the paper we are concerned entirely with the full basis $\mathcal{F}$ we simply say in what follows "continuous" instead of " $\mathcal{F}$-continuous", "major" and "minor" function instead of "F-F-major" and "F $\mathcal{F}$-minor" function and we omit the indication of the basis in the notation of the derivatives.

Theorem 5 A function $f$ is $P$-integrable on $\Delta$ if and only if it is $P_{0}$-integrable on $\Delta$. Moreover the two integrals coincide.

Proof. It is obvious that any $P_{0}$-integrable function is also $P$-integrable and that the integrals coincide.

Let $f$ be $P$-integrable over $\Delta$ and $F(I)=(P) \int_{I} f$ be its indefinite integral. We call a point $x \in \Delta$ regular if $f$ is $P_{0}$-integrable on each sufficiently small interval $I \subset \Delta$ containing $x$. Let $Q$ be the set of all non-regular points of $\Delta$. It is clear that the set $Q$ is closed and that, in view of the additivity of the $\left(P_{0}\right)$-integral, $f$ is $P_{0}$-integrable with $\left(P_{0}\right) \int_{J} f=F(J)$ on every subinterval $J$ of $\Delta$ which contains no points of $Q$.

We have to prove that $Q$ is empty. If not, then we apply Proposition 2 to $Q$ and we find a non-empty portion $T$ of $Q$ on which $f$ is Lebesgue integrable. Let $I_{0}$ be an interval such that $T=I_{0} \cap Q \neq \emptyset$. We get a contradiction if we prove that $f$ is $P_{0}$-integrable on $I_{0}$ and

$$
\left(P_{0}\right) \int_{I_{0}} f=F\left(I_{0}\right)
$$


The function $f \chi_{T}$, where $\chi_{T}$ is the characteristic function of $T$, is Lebesgue integrable and consequently $P_{0}$-integrable on $I_{0}$ (see $[8$, p. 191]), and

$$
\left(P_{0}\right) \int_{I_{0}} f \chi_{T}=(P) \int_{I_{0}} f \chi_{T}
$$

Consider on $I_{0}$ the function

$$
g=f-f \chi_{T} .
$$

It is $P$-integrable on $I_{0}, P_{0}$-integrable on each subinterval of $I_{0}$ which contains no points of $T$ and $g(x)=0$ if $x \in T$. Let

$$
G(I)=(P) \int_{I} g
$$

be the indefinite $P$-integral of $g$ on $I_{0}$.

Take a sequence $\left\{J_{j}\right\}$ of nonoverlapping intervals such that $I_{0} \backslash T=\cup_{j=1}^{\infty} J_{j}$ and each interval $J \subset I_{0} \backslash T$ is covered by a finite number of $J_{j}$. Note that any point $x$ of $I_{0} \backslash T$ belongs to no more than $2^{n}$ intervals of the sequence $\left\{J_{j}\right\}$.

Since $J_{j} \cap T=\emptyset$, we have that $g$ is $P_{0}$-integrable on $J_{j}$ and

$$
\left(P_{0}\right) \int_{J_{j}} g=G\left(J_{j}\right) \text { for each } j .
$$

Fix $\epsilon>0$, apply Corollary 4 of Proposition 3 to the function $g$ and consider a positive function $\delta$ for which

$$
V_{\delta}\left(I_{0}\right)=V\left(\mathcal{F}_{\delta}, G, T, I_{0}\right)<\frac{\epsilon}{2} .
$$

According to the definition of $P_{0}$-integral there exists for each $j=1,2, \ldots$, a continuous superadditive major function $M_{j}$ on $J_{j}$ such that

$$
0 \leq M_{j}\left(J_{j}\right)-G\left(J_{j}\right)<\frac{\epsilon}{2^{j+1}} .
$$

We define for any $J \subset I_{0}$ the function

$$
R(J)= \begin{cases}\sum_{j: J_{j} \cap J \neq \emptyset}\left(M_{j}\left(J \cap J_{j}\right)-G\left(J \cap J_{j}\right)\right) & \text { if } \quad J \backslash T \neq \emptyset, \\ 0 & \text { if } J \backslash T=\emptyset .\end{cases}
$$

By (13) and (14) we have

$$
0 \leq R\left(I_{0}\right)<\frac{\epsilon}{2}
$$


As $M_{j}$ is superadditive, it is easy to check that $R$ is also superadditive. For any interval $J \subset I_{0} \backslash T$ the sum in (14) is finite and

$$
R(J)=\sum_{j=1}^{k}\left(M_{s_{j}}\left(J \cap J_{s_{j}}\right)-G\left(J \cap J_{s_{j}}\right)\right),
$$

where $J=\cup_{j=1}^{k}\left(J \cap J_{s_{j}}\right)$.

We show that $R$ is continuous. If $x \in I_{0} \backslash T$ then the continuity of $R$ at $x$ follows from (16), the continuity of $M_{j}$ at each point of $J_{j}$ and the continuity of $G$.

Now let $x \in T$. For any $\gamma>0$ choose $j_{0}$ such that $\epsilon 2^{-j_{0}-1}<\gamma$. If an interval $J$ is chosen so small that $x \in J$ and $J \cap J_{j}=\emptyset$ for $j=1,2, \cdots, j_{0}$, then from (13) and (14) we get

$$
R(J) \leq \sum_{j=j_{0}+1}^{\infty} \frac{\epsilon}{2^{j+1}}=\frac{\epsilon}{2^{j_{0}+1}}<\gamma .
$$

This implies the continuity of $R$ at each point of $T$.

Now we show that

$$
M=G+R+V_{\delta}
$$

is a continuous major function of $g$ on $I_{0}$.

$M$ is superadditive as $G$ is additive and $R$ and $V_{\delta}$ are both superadditive.

The continuity of $M$ follows from the continuity of each term in (17) (apply Proposition 1 with $\mathcal{B}=\mathcal{F}, F=G$ and $E=T)$.

By (12), (15) and (17) we have

$$
0 \leq M\left(I_{0}\right)-G\left(I_{0}\right)<\epsilon .
$$

Now we are proving that

$$
\underline{D} M(x) \geq g(x) \text { if } x \in I_{0} .
$$

Let $x \in I_{0} \backslash T$. Then we can find $\gamma>0$ such that for all intervals $J \in$ $\mathcal{F}_{\gamma}[\{x\}]$ we have $x \in J_{s_{j}}$ if $J \cap J_{j} \neq \emptyset$, i.e. $J=\cup_{j=1}^{k}\left(J \cap J_{j}\right)$ implies $J \cap J_{j} \in$ $\mathcal{F}_{\gamma}[\{x\}], j=1,2, \cdots, k\left(1 \leq k \leq 2^{n}\right)$. For such $J,(16)$ and (17) imply

$$
M(J)=\sum_{j=1}^{k} M_{j}\left(J \cap J_{j}\right)+V_{\delta}(J) .
$$

As $M_{s_{j}} j$ is a major function on $J_{s_{j}}$ and $x \in J_{s_{j}}$, we have

$$
\underline{D} M_{j}(x) \geq g(x), \quad j=1,2, \ldots, k .
$$


Hence for any $\eta>0$ there exists $\alpha \leq \gamma$ such that

$$
\frac{M_{s_{j}}\left(J \cap J_{s_{j}}\right)}{\left|J \cap J_{s_{j}}\right|}>g(x)-\eta \text { if } J \cap J_{s_{j}} \in \mathcal{F}_{\alpha}[\{x\}], \quad j=1,2, \ldots, k
$$

Applying the obvious inequality

$$
\sum_{j} a_{j} \geq\left(\min _{j} \frac{a_{j}}{b_{j}}\right) \sum_{j} b_{j}
$$

to the case $a_{j}=M_{s_{j}}\left(J \cap J_{s_{j}}\right), b_{j}=\left|J \cap J_{s_{j}}\right|$ we get from (20) and (21)

$$
\frac{M(J)}{|J|} \geq \min _{j: J \cap J_{j} \neq \emptyset} \frac{M_{s_{j}}\left(J \cap J_{s_{j}}\right)}{\left|J \cap J_{s_{j}}\right|}>g(x)-\eta
$$

for any $J \in \mathcal{F}_{\alpha}[\{x\}]$.

Since $\eta$ is arbitrary (20) follows for $x \in I_{0} \backslash T$.

Now let $x \in T$. Then $g(x)=0$ and by (14) and (17) for any $J \in \mathcal{F}_{\delta}[\{x\}] \cap$ $\mathcal{F}_{\delta}\left(I_{0}\right)$ we have

$$
M(J) \geq G(J)+V_{\delta}(J) \geq 0
$$

and so

$$
\underline{D} M(x) \geq 0=g(x) .
$$

Hence (19) holds true for all $x \in I_{0}$ and therefore $M$ is a continuous major function of $g$ on $I_{0}$ satisfying the condition (18).

In a similar way we can construct a continuous minor function $m$ of $g$ satisfying the inequality

$$
0 \leq G\left(I_{0}\right)-m\left(I_{0}\right)<\epsilon .
$$

This proves that $g$ is $P_{0}$-integrable on $I_{0}$ with $G(J)$ being the indefinite $P_{0^{-}}$ integral of $g$ on $I_{0}$.

Then (9), (10) and (11) imply that $f$ is $P_{0}$-integrable on $I_{0}$ and (9) holds true, giving the desired contradiction.

\section{References}

[1] Z. Buczolich, Henstock integrable functions are Lebesgue integrable on a portion, Proc. Amer. Math. Soc., 111(1) (1991), 127-129.

[2] P. S. Bullen et al.(ed), New integrals, Lecture Notes in Math., 1419, Springer-Verlag, 1990. 
[3] Z. T. Gong, On a problem of Skvortsov involving the Perron Integral, Real Analysis Exchange, 17(2) (1991-92), 748-750.

[4] M. de Guzman, Differentiation of Integrals in $\mathbb{R}^{n}$, Lecture Notes in Math., 481, Springer Verlag, 1975.

[5] S. Lu, On the construction of major and minor functions, Journal of Mathem. Study, 27(1) (1991), 124-126.

[6] M. P. Navarro and V. A. Skvortsov, On n-dimensional Perron integral, South East Asia Math. Bull., to appear.

[7] K. M. Ostaszewski, Henstock integration in the plane, Memoirs Amer. Math. Soc., Providence, 353, 1986.

[8] S. Saks, Theory of the integral, Dover, New York, 1964.

[9] V. A. Skvortsov, Continuity of $\delta$-variation and construction of continuous major and minor functions for the Perron integral, Real Analysis Exchange, 21(1), 1995.

[10] B. S. Thomson, Derivates of intervals functions, Memoirs Amer. Math. Soc., Providence, 452, 1991. 